may be ingeniously overcome by coating the resin with an indigestible material which is degradable by bacteria so that the resin is dispersed only where it is needed, in the colon. On p 1315 Einar Krag's group in Copenhagen shows that such tablets work well and are free of the undesired effects. Should tablets of this type be marketed they will provide a useful alternative to standard antidiarrhoeal drugs in some patients with "functional diarrhoea."

K W HeatoN

Reader in Medicine,

Bristol Royal Infirmary,

Bristol BS2 8HW

1 Thompson WG, Heaton $\mathrm{KW}$. Functional bowel disorders in apparently healthy people. Gastroenterology 1980;79:283-8.

Harvey RF, Salih SY, Read AE. Organic and functional disorders in 2000 gastroenterology outpatients. Lancet 1983; : $632-4$.

Hillman LC, Stace NH, Fisher A, Pomare EW. Dietary intakes and stool characteristics of patients with the irritable bowel syndrome. Am $f$ Clin Nutr 1982;36:626-9.

Cann PA, Read NW, Brown C, Hobson N, Holdsworth CD. Irritable bowel syndrome: relationship of

Jones VA, McLaughlan P, Shorthouse M, Workman E, Hunter JO. Food intolerance: a major factor in the pathogenesis of irritable bowel syndrome. Lancet 1982;ii:1115-7.

factor in the pathogenesis of irritable bowel syndrome. Lancet 1982;ii:1115-7. 3rd ed. Philadelphia: Saunders, 1983:257-80.

Heaton KW, Morris JS. Bitter humour: the development of ideas about bile salts. $\mathcal{F} R$ Coll Physicians Lond 6:83-97.

Forth W, Rummel W, Glasner $H$. Zur resorptionshemmenden Wirkung von Gallensäuren. Naunyn Schmiedebergs Arch Pharmacol 1966;254:364-80.

Mekhiian HS, Phillips SF, Hofmann AF. Colonic secretion of water and electrolytes induced by bile acids: perfusion studies in man. $\mathcal{J}$ Clin Invest 1971;50:1569-77

Taylor I, Basu P, Hammond P, Darby C, Flynn M. Effect of bile acid perfusion on colonic motor function in patients with the irritable colon syndrome. Gut 1980;21:843-7.

bersen L, Arnfred T, Hess Thaysen E. Rapid screening of increased bile acid deconjugation and bile acid malabsorption by
Gastroenterol 1973;8:665-72.

12 Thaysen EH, Pedersen L. Idiopathic bile acid cartharsis. Gut 1976;17:965-70.

13 Merrick MV, Eastwood MA, Ford MJ. Is bile acid malabsorption underdiagnosed? An evaluation of accuracy of diagnosis by measurement of SeHCAT retention. Br Med $\mathcal{J}$ 1985;290:665-8.

Cann PA, Read NW, Holdsworth CD, Barends D. Role of loperamide and placebo in management of irritable bowel syndrome (IBS). Dig Dis Sci 1984;29:239-47.

\section{Pneumonia in the acquired immune deficiency syndrome}

To date there have been 130 cases of the acquired immune deficiency syndrome (AIDS) in Britain. Although this is far short of the 7000 diagnosed in the United States, the projection suggests as many as 400 cases by the end of 1985 : the number is doubling every six months. ${ }^{1}$ Most cases in Britain have presented to departments of genitourinary medicine in central London because these departments have many homosexual men as patients. As the disease spreads among the homosexual community patients with AIDS may be expected to present to their general practitioners throughout the country rather than directly to a London hospital. In turn, because AIDS is a multisystem disease, the general practitioner may refer the patient to a respiratory physician, gastroenterologist, neurologist, general surgeon, dermatologist, or genitourinary physician. Sooner or later, however, most patients develop respiratory problems.

AIDS is thought to be caused by the retrovirus human $T$ cell lymphotropic virus III/lymphadenopathy associated virus (HTLV-III/LAV), which specifically attacks and destroys OKT4 (helper) T cells..$^{2-4}$ Virtually all patients with established AIDS have antibodies to HTLV-III in their serum. ${ }^{5}$ This test is not diagnostic, however, because of the high positivity in asymptomatic homosexuals. Three quarters of all cases of AIDS occur in homosexual or bisexual men. Other smaller groups at risk are intravenous drug abusers; haemophiliacs given commercial factor VIII; patients transfused with infected donor blood; the female sexual partners of male intravenous drug abusers, bisexual men, and haemophiliacs; babies of infected mothers; and some patients from central Africa and the West Indies. Haitians account for $3 \%$ of the cases in the United States but are rarely seen in Britain. About $4 \%$ of cases do not fit into any of these groups..$^{6-8}$

An acute pneumonic illness with cough, breathlessness, and fever may be the presenting feature of AIDS, but nearly always there is a preceding history of several months' ill health characterised by loss of weight, intermittent or prolonged fever, and malaise. In our experience some patients have been well informed and aware of the possible meaning of their symptoms. They have volunteered that they believed they might have AIDS. This is not always the case, however, particularly in those who deny their sexual orientation. The pneumonia may or may not occur in association with other features of AIDS such as generalised lymphadenopathy, Kaposi's sarcoma, or oropharyngeal candidiasis.

The organism most commonly responsible for AIDS related pneumonia is the multiflagellate protozoa Pneumocystis carinii, which accounted for $373 / 441$ (85\%) cases of pneumonia in one large series from the United States ${ }^{9}$ and a similar proportion of the cases seen in Britain to date. ${ }^{10}$ Pneumonia caused by this organism is usually associated with an insidious but progressive dry cough and increasing breathlessness, usually over one or two weeks but sometimes up to eight weeks. ${ }^{11}$ Fever is normally present but is not striking. On physical examination typically there are a few bilateral scattered crackles over the affected lobes, but the chest may sound clear in the presence of definite infection. The chest radiograph characteristically shows diffuse bilateral alveolar or interstitial shadowing, but it may be normal in a few cases (probably those seen very early in the disease). In all cases, however, there is hypoxaemia, which may be profound, especially if the patient ignores the warning symptoms, in which case he may present in hypoxic respiratory failure.

Even in early pneumonia due to $P$ carinii the corrected transfer factor for carbon monoxide is reduced, but interpretation of this finding may be difficult if there are other possible causes such as intravenous drug abuse or smoking. The gallium-67 lung scan is positive, but in one series there were also many false positives. ${ }^{9}$ In practice these additional tests are required only when the chest $x$ ray film is normal in a patient whose clinical features suggest $P$ carinii pneumonia. One additional test usually suffices. We use the $\mathrm{PaO}_{2}$ because it provides a rapid answer and the apparatus can be sterilised. Great care should be taken in obtaining the sample. ${ }^{12}$

Other opportunistic organisms which have been associated with AIDS related pneumonia in the United States include Mycobacterium avium intracellulare and cytomegalovirus. Whereas cytomegalovirus has frequently been found in Britain, $M$ avium intracellulare has not. It must also be remembered that patients with AIDS are at increased risk of developing more conventional pneumonias with organisms such as Streptococcus pneumoniae. ${ }^{9}$ In these circumstances, however, the patient is more acutely ill with the sudden onset of high fever, purulent sputum, and signs of consolidation in the chest and with radiological evidence of lobar or segmental consolidation. Multiple organisms are detected in many patients with AIDS. In the combined American experience 118 of 373 patients with $P$ carinii pneumonia had coexisting pathogens including legionella, $M$ tuberculosis, aspergillus, cryptococcus, herpes simplex, and 
toxoplasma as well as the organisms already described. In addition Kaposi's sarcoma affects the lung in about $8 \%$ of patients with AIDS. ${ }^{9}$

Ideally the causative organism of any pneumonia should be identified in order to select rational treatment. In patients suspected of having AIDS the diagnosis may not be possible until an opportunistic organism has been identified. ${ }^{13} P$ carinii may be identified morphologically only in stained specimens of bronchoalveolar lavage or of alveolar tissue obtained by transbronchial, percutaneous needle, or open lung biopsy. So far as $P$ carinii pneumonia is concerned the diagnostic yield from fibreoptic bronchoscopy is high; transbronchial biopsy seems to give better results (95\% yield) than bronchoalveolar lavage (79\%) or brushing (39\%). ${ }^{9}$ With other bacterial and viral infections bronchoalveolar lavage enables the organisms to be cultured, and $M$ avium intracellulare and cytomegalovirus are usually detected in this way. Thus the combination of transbronchial biopsy and bronchoalveolar lavage is ideal. ${ }^{14}$ The diagnosis of Kaposi's sarcoma usually requires open lung biopsy. ${ }^{14}$

Some clinicians may question whether such aggressive investigation is really necessary. At a meeting held in London at the end of last year there was protracted discussion for and against ( $\mathrm{N} \mathrm{McI} \mathrm{Johnson,} \mathrm{convener).} \mathrm{On} \mathrm{the}$ one hand, the procedures were seen to be necessary to make a precise diagnosis of the cause of the pneumonia-and indeed to establish the diagnosis of AIDS, with all that that implies to the patient and his friends and relatives. On the other hand, the risks to the operators and assistants were unknown, and results had only limited effects on clinical management. Quite probably as physicians' experience increases they will choose the most likely pathogen on clinical and radiological grounds and treat accordingly, reserving transbronchial biopsy and bronchoalveolar lavage for patients who fail to respond. Since, however, many patients are likely to have multiple infecting organisms the initial choice of antibiotics is likely to be difficult. Moreover, the length of treatment required for $P$ carinii pneumonia and the toxicity of the drugs used provide further arguments against treatment without definite proof of the diagnosis. The question of repeat bronchoscopies for recurrence of pneumonic illnesses is also unsolved; if the patient has suffered from a bacterial pneumonia repeat transbronchial biopsy is indicated to identify the new pathogen, but in any patient who has had one episode of $P$ carinii pneumonia repeat lung biopsy specimens may contain $P$ carinii even after a good clinical response to the initial episode. ${ }^{11} 15$

Conventional pneumonias should be treated along normal lines with oxygen, physiotherapy, and broad spectrum antibiotics. The treatment of choice for $P$ carinii pneumonia is with high doses of intravenous co-trimoxazole (trimethoprim $20 \mathrm{mg} / \mathrm{kg}$ a day in divided doses) continued for three weeks. Drug toxicity is common at this dose but only rarely are the symptoms serious enough to force withdrawal of the drug. One important risk is serious thrombocytopenia, but folinic acid may prevent this. ${ }^{16}$ When serious toxic reactions occur or in cases of allergy to sulphonamides the alternative treatment is pentamidine ( $4 \mathrm{mg} / \mathrm{kg}$ a day intramuscularly). This treatment is frequently associated with a rise in the blood concentrations of urea and creatinine; these return to normal if the drug is stopped when the rise is first noted. Local reactions may occur at the injection site. ${ }^{17}$ In the United States when patients needed to be transferred from co-trimoxazole to pentamidine because of drug toxicity the prognosis was still good (100\% survival), but when the change was made because of failure to respond to treatment the outlook was poor ( $89 \%$ mortality). ${ }^{9}$

The mortality from a first attack of $P$ carinii pneumonia is about one third. ${ }^{9}$ In those who survive it may be beneficial to give prophylaxis with either low dose co-trimoxazole or Fansidar to prevent recurrence-a common phenomenon, because $P$ carinii is often found in repeat lung biopsy specimens even after the patient has made a clinical and radiological recovery from an attack after three weeks of intravenous treatment. ${ }^{18}$ Recurrences of $P$ carinii are less responsive to co-trimoxazole and the outcome poorer (by this time the patient often shows signs of general clinical deterioration), and pentamidine may be the best initial treatment for a second attack. ${ }^{9}$ There is no evidence to support the use of the combination of pentamidine and cotrimoxazole in $P$ carinii pneumonia.

Patients with atypical mycobacterial infection should be given antituberculous treatment, although the organisms are more resistant than $M$ tuberculosis, and the prognosis is poor. Ansamycin and clofazimine are two drugs at present under trial in the United States. ${ }^{9}$ Cytomegalovirus pneumonias do not respond to antiviral agents.

How should the patient be nursed? HTLV-III may be isolated from blood and saliva as well as semen, ${ }^{3}$ and these secretions should be regarded as sources of infection. The infectivity of the virus and the numbers of people infected with HTLV-III who go on to develop AIDS are unknown. The risk of cross infection to medical staff is thought to be small, but care is obviously required. To date we have been nursing these patients within side wards in our acute general hospital using the same precautions as for hepatitis B infection. ${ }^{19}$ Bronchoscopies have been performed with the operator wearing a non-absorbent gown, mask, hat, gloves, and goggles (to prevent conjunctival spread) and using a separate fibreoptic bronchoscope which has been sterilised after use in glutaraldehyde followed by ethylene oxide. This sterilisation procedure (which takes four days) may be overelaborate as it is now thought HTLV-III is killed by heat, formaldehyde, glutaraldehyde, alcohol, and hypochlorite ( $\mathrm{R}$ Tedder, personal communication) but it seems best to err on the side of caution. All specimens obtained from such patients must be suitably labelled and the laboratories warned of the potential risk as suggested by the Advisory Committee on Dangerous Pathogens. ${ }^{19}$

Should these patients be treated in a single centre or within an infectious diseases unit? The main problems with this approach are the rising numbers expected of patients with AIDS, the duration of their hospital admissions, and the complex investigations they need.

What is the overall prognosis? In over 1000 cases in New York the median cumulative survival of patients with AIDS with Kaposi's sarcoma alone was 125 weeks, in those with Kaposi's sarcoma and pneumocystis pneumonia 61 weeks, and for those presenting with pneumocystis pneumonia alone 35 weeks. $^{20}$ Thus while one or two episodes of pneumonia in an individual patient may be treated successfully if referral is early enough, until treatment for the underlying primary disease is available we are prolonging life for a few months rather than offering a cure. The quality of life is variable: in one series $40 \%$ of patients spent more than half the time from diagnosis to death in hospital. ${ }^{21}$ If the patient with $P$ carinii is referred too late or fails to respond to initial treatment progressive hypoxic respiratory failure ensues, from which recovery is rare (14\%), even with artificial ventilation in an intensive care unit. ${ }^{9}$ All the patients who presented to us with pneumonia up to June 
1984 were dead by the end of the year despite good initial survival figures.

Senior Lecturer in Medicine,

NORMAN MCI JOHNSON

Middlesex Hospital Medical School,

London WIN 8AA

1 Ebbesen P, Biggar RJ, Melbye M. AIDS in Europe. Br Med $\mathcal{f}$ 1983;287:1324-5.

2 Barre-Sinoussi F, Chermann JC, Rey F, et al. Isolation of a T-lymphotropic retrovirus from a patient at risk from the acquired immune deficiency syndrome (AIDS). Science 1983;220 patient

3 Broder S, Gallo RC. A pathogenic retrovirus (HTLV-III) linked to AIDS. $N$ Engl f Med 1984;311:1292-7.

4 Seligmann $M$, Chess L, Fahey JL, et al. AIDS-an immunologic re-evaluation. $N$ Engl f Med 1984:311:1287-97.

5 Cheingsong-Popov R, Weiss RA, Dalgleish A, et al. Prevalence of antibody to human T-lymphotropic virus type III in AIDS and AIDS-risk patients in Britain. Lancet 1984;ii: 477-80

6 Adler MLD, Weller IVD. AIDS-sense not fear. Br Med f 1984;288:1177-8.

7 Clumeck N, Mascart-Lemone F, DeMaubeuge J, Brenez D, Marcelis L. Acquired immune deficiency syndrome in black Africans. Lancet 1983;i:642.

8 Center for Disease Control. Update-acquired immunodeficiency syndrome (AIDS)—United States. $M M W R$ 1984;33:661-4

9 Murray JF, Felton CP, Garay SM, et al. Pulmonary complications of the acquired immunodeficiency syndrome. Report of a National Heart, Lung and Blood Institute workshop. $N$ Engl f Med 1984;310:1682-8.

10 Swinburn CR, Pozniak AL, Banks RA, Teal AJ, Johnson NMcI. Early experience and difficulties with bronchoalveolar lavage and transbronchial biopsy in the diagnosis of AIDS difficulties with bronchoalveolar lavage and transbronch
associated pneumonia in Britain. Thorax 1985;40:166-70.

11 Engelberg LA, Lerner CW, Tapper ML. Clinical features of pneumocystis pneumonia in the acquired immune deficiency syndrome. Am Rev Respir Dis 1984;130:689-94.

12 Anonymous. Needlestick transmission of HTLV III from a patient infected in Africa [Editorial]. Lancet 1984;ii:1376-7.

13 Center for Disease Control. Update on acquired immunodeficiency syndrome (AIDS)-United States. MMWR 1982;31:507-8.

14 Stover DE, White DA, Romano PA, Gellene RA. Diagnosis of pulmonary disease in acquired immune deficiency syndrome (AIDS)-role of bronchoscopy and bronchoalveolar lavage. $A m$ Rev Respir Dis 1984;130:659-62.

15 Rosen MJ, Teirstein AS, Chuang MT, Brown LK. Response to therapy of Pneumocystis carinii pneumonia in patient with AIDS. Chest 1983:84:347.

16 Jaffe HS, Abrams DI, Ammann AJ, Lewis BJ, Golden JA. Complications of co-trimoxazole in treatment of AIDS-associated Pneumocystis carinii penumonia in homosexual men. Lancet treatment of AIDS

17 Hughes WT, Feldman S, Chaudhary SC, Ossi MJ, Cox F, Sanyal SK. Comparison of pentamidine isethionate and trimethoprim-sulfamethoxazole in the treatment of Pneumocystis carinii pneumonia. F Pediatr 1978;92:285-91.

18 Gottlieb MS, Knight S, Mitsuyasu R, Weisman J, Roth M, Young LS. Prophylaxis of Pneumocystis carinii infection in AIDS with pyrimethamine sulfadoxine. Lancet 1984;ii: 398-9.

19 Conte JE, Hadley WK, Sande M, University of California, San Francisco Task Force on the Acquired Immune Deficiency Syndrome. Infection control guidelines for patients with the acquired immune deficiency syndrome (AIDS). $N$ Engl f Med 1983;309:740-4.

19a Advisory Committee on Dangerous Pathogens. Acquired immune deficiency syndrome (AIDS) interim guidelines. London: DHSS, 1984. $(\mathrm{HC}(85) 1$.)

20 Rivin BE, Monroe JM, Hubschman BP, Thomas PA. AIDS outcome: a first follow-up. N Engl f Med 1984;311:857.

\section{Occult advanced cervical cancer}

Early, apparently localised carcinoma of the cervix is a common problem in gynaecological practice. Nearly 4000 new cases of cervical cancer were diagnosed in England and Wales in $1978^{1}$ and about half of these presented at an early stage. ${ }^{23}$ The International Federation of Gynaecologists and Obstetricians allocates patients with pathologically proved invasive cervical carcinoma clinically limited to the cervix to stage IB. The prognosis for these patients is good with reported five year survival rates of $75-90 \% .{ }^{4}$ Radiotherapy or surgery or both are used in treatment, with no method having been shown to have a consistent advantage.

We believe that within that group of patients with apparently localised disease there is a subgroup with a considerably poorer prognosis. These patients in reality have microscopic spread of disease beyond the cervix. By definition, staging is clinical and based on the results of examination and radiological investigations. When clinical staging is compared with the findings at laparotomy, however, over a quarter of the patients assigned to stage IB prove to have been understaged. ${ }^{5}$ Step sectioning of pelvic lymph nodes is superior to the more commonly practised random sectioning, ${ }^{6}$ and it is not difficult to imagine that the same process applied to paracervical tissue would show some patients with microscopic extension of disease beyond the cervix. At present micrometastases are not detected, but they will lead to recurrence after apparently successful and adequate surgery.

Patients who have extension of their disease beyond the pelvis have a prognosis dependent on the extent of this spread. Survival rates range from $50 \%$ for those patients with spread to one group of nodes unilaterally, ${ }^{7}$ to virtually nil for patients with macroscopic spread to the para-aortic nodes. ${ }^{8}$ Increasing numbers of nodes and increasing numbers of groups of nodes affected, unilateral spread, and cephalad spread of affected nodes all confer a poorer prognosis.

Laparotomy provides the most accurate basis for predicting the outcome in individual patients. Three other factors influence the risk of unrecognised extension of the tumour beyond the cervix. Firstly, patients with tumours over $4 \mathrm{~cm}$ diameter have a less than evens chance of five year survival. ${ }^{9}$ Secondly, patients with disease affecting more than two thirds of the thickness of the cervix have recurrence rates of $58-85 \% .^{10}$ The third factor is the degree of differentiation, which has correlated with outcome in most studies, anaplastic tumours having a poorer prognosis than the well differentiated ones." This is especially true of the keratinising small cell types. ${ }^{12}$

Considerable controversy surrounds the clinical course of cervical carcinoma in young women (below the 35-40 age group). Early studies suggested that the survival in this group was no different from that of those over $35 .{ }^{13}$ Recent experience in Britain, however, has suggested that this is not the case. ${ }^{14}$ Patients presenting under the age of 40 with stage IB carcinoma of the cervix have a five year survival below $50 \%$. One third have metastases in the pelvic nodes, and well differentiated keratinising tumours occur in only $6 \%$, as against $25 \%$ in those aged over 40 (J M Monaghan, unpublished data). Overall, the patient at highest risk of recurrence has one or more of the following features: youth, a large, poorly differentiated tumour affecting most of the cervix, and diseased nodes. The same pattern is seen in patients with stage IB adenocarcinoma of the cervix. ${ }^{15}$

What can be done for these patients? The conventional treatment for patients with spread of disease to lymph nodes is surgery and adjuvant radiotherapy. Unfortunately, no randomised or prospective trial has been performed on the efficacy of this management-but published retrospective data suggest no survival advantage for patients so treated..$^{26916-20}$ This may be because the tumour is outside the treatment field or the dose of radiation that can be delivered to the treatment field is insufficient.

A systemic approach seems, therefore, to be more logical, but chemotherapy for carcinoma of the cervix has not received much attention. Studies in advanced or recurrent disease have shown response rates of $10-60 \%$ with a range of established anticancer drugs either singly or in combination. ${ }^{21}$ Until the incorporation of cis-diamminedichloroplatinum II (cisplatin) into the combinations these responses had universally been short lived. Two recent reports, however, have suggested long term complete response rates of $20-30 \%$ with combinations of cisplatin, bleomycin, and vinblastine with or without mitomycin C. ${ }^{22}$ Partial responses were seen in a further $14-43 \% .{ }^{18} 19$ These results approach those achieved in chemotherapy of ovarian carcinoma.

Only one trial has been reported of adjuvant chemotherapy in patients with early disease treated by surgery..$^{10}$ In a nonrandomised but prospective study using either a single antifolate or alkylating agent a survival advantage was shown 